\title{
What's shaking? Molecular-dynamics simulations of X-ray diffraction from crystalline proteins.
}

\author{
M WALL ${ }^{1}$ \\ ${ }^{1}$ LOS ALAMOS NATIONAL LABORATORY, LOS ALAMOS, NM \\ mewall@lanl.gov
}

$\mathrm{X}$-ray diffraction data contain rich information about collective motions in protein crystals. Extracting this information has been historically difficult, but advances in data collection and computing are enabling studies of Xray diffraction from protein crystals using molecular-dynamics (MD) simulations. This talk will examine recent results with a special focus on crystallographic water structure and diffuse scattering -- intensity beneath and away from the Bragg peaks in diffraction images. These results indicate that MD simulations can add realistic descriptions of motions at atomic detail that agree substantially with protein crystallography data. At the same time, limitations in the accuracy of the simulations suggest a path forward for improving MD models and force fields.

Acta Cryst. (2020). A76, a104 